MATEC Web of Conferences 44, 01047 (2016)

DOI: $10.1051 /$ matecconf/20164401047

(C) Owned by the authors, published by EDP Sciences, 2016

\title{
The Applied Research on Computer Aided Design in Art Design
}

\author{
Yong Min Cui ${ }^{1}$, Xin Guang Ren ${ }^{2, a}$ \\ ${ }^{1}$ Rongcheng College, Harbin University of Science and Technology, Rongcheng; Shandong, China \\ ${ }^{2}$ Rongcheng College, Harbin University of Science and Technology, Rongcheng; Shandong, China
}

\begin{abstract}
Aiming at constant improvement of computer technology development, the paper proposes the specific applications and prospects of Computer Aided Design(CAD) in the art design field by considering applied research background of CAD in art design, investigating and analyzing superiority and status of CAD, and reinforcing important significance of CAD on art design development. Only to create the CAD continuously and promote applied skills of CAD in art design field can make art design assist CAD to development continuously and satisfy pursuits of material progress and spirit civilization.
\end{abstract}

In today's society with rapid development of technology and continuous improvement of computer technology, computer technology has been applied to numerous fields and also has been widely applied in art design. Computer technology is equipped with sufficient superiority in price and performance, owing to continuous development of network communication technology, so computer technology performs a huge potential in the art design field. Speaking of computer design, Hearst Randolph once said that "computers impact thinking modes of users. It has compulsive demands for logical analysis, accurate formula and quantitative expressions". Computers can help people to have rationalized thinking, analyze and process all kinds of logical information rapidly. Thus, art design is established on the basis of modern science to constitute assisted design, and such a mode is the feature of modern art design and also opens up a new research field for us to engage in art design career.

\section{Features of Computer-assisted Art Design}

\subsection{Superiority of Computer-assisted Art Design}

With the deep development of computer technology, the status of CAD in art design field is increasingly important. For example, industrial design, advertising design, webpage design and animation design, etc., various art design fields concentrate on embodying ability of CAD. One of prominent advantages of computers is to provide greater degree of freedom for designers, who can apply computers to switch and combine pictures at will, as well as attempt various different configurations to create unexpected effects. In this way, it not only can seek for the most excellent and satisfactory works, but also stimulate creative inspiration of designers simultaneously. Secondly, applications of computers improve design precision and speed effectively, reduce labor intensity of designers, and reduce tedious process of some complicated design works. For instance, CAD system can make data of interior design become accurate and reliable and avoid from unnecessary losses caused by errors in the construction project. For some animation design, computers can give simulation preview, and virtuality of real technology makes design performance intuitive and vivid, so that animation drawing will be more accurate. Nowadays, with the constant occurrence of computer three-dimensional software, designers generally can perform their creativity and inspiration by using computers. For example, they draw a three-dimensional spatial model to express shape, structure and size of design products, stimulate color and texture of product surface under the lighting, shorten deign time greatly, and promote design quality effectively. Another advantage of computer technology refers to user-friendly control. It can bring great convenience to designers and change malpractice of time-consuming handwork drawing effectively. In the design working process, it inevitably will appear more or less problems. A simple cancel command of computers can change wrong steps and offer largely free alternation space to designers.

In addition, many computer-assisted manufacturing operations can't be handled by traditional handicraft. For example, crystal laser art engraving uses a model with good three-dimensional software design and graves by using a laser engraving machine to generate complicated, miraculous and distinctive three-dimensional laser engraving graphs. The development of rapid science and technology brings new revolution in art design, and this is the new beginning of better combining technology and art.

\footnotetext{
a Xin Guang Ren:renxinguang12@163.com
} 


\subsection{Status of CAD}

At present, software development of CAD changes quickly, and functions are increasingly strong and become widely in various fields. It has moved towards life and work of humans gradually and unconsciously and also becomes an indispensable part in the design industry. Therefore, it almost can say that all design industries apply CAD, commonly including CAD in the building field, which generally is embodied in two forms: on the one hand, it is about production software of effect pictures represented by 3DStudioVIZ to make houses or stimulate interior finishing effects. On the other hand, it refers to $\mathrm{CAD}$ in interior design represented by AutoCAD and can complete accurate construction drawing design and structure analysis.

As for the CAD in print advertisement, modern printing technology has changed inflexible and typing, etc., traditional technology completely to finish through computer operation from design to output. Under the assistance of Photoshop and InDesign, etc., software, people have already realized the working mode that what you see is what you get. Our thinking can outline rudiment on a computer easily. After repeated modification, perfection and special-effect combination, these productions will be better expressed.

\subsection{Significance of CAD on Art Design}

Art design is a kind of technological means. It refers to inartistic (especially for non-pure artistic) material product design and also has certain aesthetic property. Art design is an important constituent part of human civilization and mainly constructs material and spiritual civilization. The art design aesthetics still is the bridge to communicate with two major civilized fields, while main research object of technological aesthetics can be objected only by virtue of object production. From the perspective of social meaning, art design always makes progress with technological level synchronously. Design objects, means, thinking or even designers have huge changes due to powerful intervention of technology. Art design depends on technology, so the relationship between computer technology and art design is not opposite, but is interdependent and indivisible.

Computer technology performs art design, while art design needs computer technology to serve for modern market. The core of art design is to innovate and create. $\mathrm{CAD}$ is the most advanced and effective tool in our design, is another way of exploiting design thinking and also is an essential advanced brush in modern design. Designers are design subjects, while computers are tools. As a result, in the process of design creation, it not only cultivates innovation consciousness consciously, enriches the ability of artistic appreciation, exploits and enriches design methods and innovation, and improves artistic culture and design ability, but also reinforces the application ability of CAD. Art design has bright fashionability, so it means that it need to integrate knowledge of modern science technology into it and from synchronous development of times culture and social knowledge.

\section{Applications of Computer Technology in the Art Design Field}

In recent years, the applied range of computer technology is increasingly extensive in the art design field, such as building art design, animation design, and graphic art design, which are contributed to CAD.

\subsection{Applications of CAD in the Building Art Design}

CAD has widely extensive applications in the building art design and mainly reflects in the following two aspects: First of all, it refers to design building effect pictures. When designing building effect pictures, it needs to use $\mathrm{CAD}$, so it not only can shorten design time effectively, but also can perfect design effects and improve goodness of fit between designed buildings and building effect pictures and viewing value of building effect pictures. Secondly, it refers to animation design. By virtue of CAD, it can present effects of buildings vertically in the form of three-dimensional animation, so people can observe achievements after completing construction of buildings intuitively. Recently, with the rapid development of social economy and continuous development of technology, art design of buildings also applies CAD greatly and improves artistic effects of buildings.

\subsection{Applications of CAD in the Animation Design}

With the constant development of social economy, the national investments of cultural industry are enlarged increasingly, especially for recent years. Thus, the nation also enlarges the supporting strength of domestic animation industries, such as Boonic Bears, and Radiant and Wolf, etc., which are deeply welcomed by the public. The animation art gives full play to advantages of CAD. In other words, animation industry is arisen with the development of computer technology. The future development of animation industry also must depend on computer industry, For example, by using computer technology, three-dimensional animation is made. In three-dimensional animation, it can set up many threedimensional figures, animals, scenes, trees and houses, or even sea waves, sands, blues sky and white cloud etc., which have higher authenticity. In the animation art design, designers can make abundant and colorful animation in line with demands and by virtue of computer technology and computer software. Meanwhile, it is also beneficial to continuous development of animation artistic industry.

The constant deepness of CAD has already exceeded the range of easy production internationally. It will be widely applied to television ads and film/television film titles. Due to integration of CAD, techniques, which can't be performed in the past, also can be realized. For instance, Jurassic Park, which has broken box office in America, has been made by special effects using computer-assisted animation technology. With the publication of excellent films and television programs, such as Star Wars, it can be seen everything by using 
computer animation design technology to make advertising production. Thus, reliable computer technology and design software with powerful functions can image and develop in the limited thinking space by designers.

\subsection{Applications of CAD in the Animation Design}

In the art design field, graphic design is a basic art major, so if CAD wants to fully being applied to building art design and animation design, it must has a wide application in graphic design. Using computer technology in graphic design not only can process graphic images, but also can change the way of graphic design. In the past, the production process of graphic design is very completed, while after using CAD, it can realize systematization and simplification of graphic design. Moreover, design effects and efficiency also will be very prominent. For example, when designing graphic images, computer graph and image processing can be used to transit, incise and process lots of graphic pictures, so as to create an extremely fantastic image world and create more refinement of graphic design images. The visual impact goes without saying and fully satisfies aesthetic demands of the public.

\section{The Development Tendency of CAD in the Art Design}

\subsection{Combination of Art Design and Multidisciplinary}

Differing from pure fine arts, art design is developed with the development of social economy and technology and is the intersection and integration of technology and aesthetics. The core of art design is "people first", so designers not only should have good foundation of fine arts, but also know about knowledge in multiple aspects, such as knowledge of materials, psychology and economics. CAD also should perform in this way. As for webpage design, it not only requires designers to have certain foundation of fine arts, but also requires designers to have the ability to apply network design language to program. This is a necessary skill to develop dynamic and interactive webpage. For another example, packing design should know about features of different packing materials and characteristics of different packing structure.

\subsection{Development, Professional and Intelligence of Design Software}

At present, the reason for surviving mainstream design software mainly benefits from development. For example, over 10 companies develops plug-in filter for photoshop, so special effects and corresponding applications of filters obtained by several changes can be completed in a time. For another example, AUTOCAD provides ADS, VBA, Java and Visuallisp, etc., multiple secondary development languages, so commercial corporations can use corresponding language development based on the plug-in platform of AUTOCAD, and designers also can use secondary development languages to complete effects which are hard to reach by using traditional computer design methods. Meanwhile, MAYA, etc., some largescale professional dimensional design software also has been spread to the domestic.

In order to make artists and designers to accept the tool- $\mathrm{CAD}$, design software also become increasingly intelligent. Painter software stimulates features of a great number of traditional tools and it lengthens the traditional painting experience. In the Painter software, plenty of tools of imitating traditional brushwork and various painting materials can stimulate oil painting, watercolor and traditional Chinese painting, etc., effects easily. Moreover, it is sensitive to texture of painting materials and drawing of designers, so artworks are rich in expressive force.

To sum up, the paper mainly analyzes and discusses art design and applications of CAD simply. The research finds that applying $\mathrm{CAD}$ in the art design field is beneficial to ensure quality of art design and improve art design level. Moreover, it is good for designers to apply more modern information technology, improve effects of art design, enhance market competitiveness, and promote constant development of art design industry.

\section{References}

[1] Muzi Li, The Study on CAD in Decorative Design [D], Harbin Normal University, 2010.6

[2] Wei Tan, Discussion on CAD and Art Design [J], Teaching Research, 2008(9)

[3] Yongning Zhang, Dongsheng Chen and Xiangning Zhang, Status and Prospect of CAD [J], 2003, (9);

[4] Xiaochuan Fu, Applied Research of CAD in Landscape Performance [D], Northwest Agriculture \& Forestry University, 2010.11;

[5] Yanhua Li, Applications and Exploration of CAD in Design Construction [J], Computer Knowledge and Technology, 2015(3);

[6] Jiming Wang, Applied Research on CAD [J], Science and Innovation, 2014(21);

[7] Yang Yang, Development and Applications of CAD

[J], Information System Engineering, 2014(2). 